\title{
Comment on Jia et al.: Three dimensional CT evaluation of femoral neck anteversion, acetabular anteversion and combined anteversion in unilateral DDH in an early walking age group
}

\author{
Aditya Krishna Mootha $\cdot$ Raghav Saini
}

Received: 5 March 2012 / Accepted: 13 April 2012 / Published online: 3 May 2012

(C) Springer-Verlag 2012

With great interest we read the article, "Three dimensional $\mathrm{CT}$ evaluation of femoral neck anteversion, acetabular anteversion and combined anteversion in unilateral DDH in an early walking age group" by Jia et al. [1], published in your esteemed journal International Orthopedics. First of all, we would like to congratulate the authors for an excellent study. However, we would like to raise a few points which need further clarification. The analysis of the data showed no statistically significant difference between dislocated and normal hips in relation to FA ( $p$-value 0.071) (Table 1). But subgroup analysis showed a statistically significant difference in the Tonnis IV group ( $p$-value 0.028 ) (Table 2). The sample size is not so large (hence non parametric tests applied for statistical analysis), so that in such conditions with further sub group analysis, there is a chance of statistical exaggeration of the difference. If we critically look at the FA (dislocated side) in the various subgroups of Tonnis II $\left(32.53 \pm 6.86^{\circ}\right)$, Tonnis III $\left(34.79 \pm 7.02^{\circ}\right)$ and Tonnis IV $\left(35.97 \pm 10.24^{\circ}\right)$, the difference among these groups might not be significant. Hence we feel that the authors' recommendation that femoral derotation may be needed if the $\mathrm{CA}$ is above $62^{\circ}$ holds for all cases irrespective of Tonnis grade, as this might also happen in Tonnis grades II or III. At the same time we are eager to know the results of the surgery in these patients from the author, as this will further strengthen their views. In our experience of 15 cases, there was no need for femoral derotation in any of them [2]. As DDH is a condition where acquiring a huge sample size is difficult we need many more studies including preoperative evaluation, intraoperative assessment and postoperative radiological reassessments.

\section{References}

1. Jia JY, Li LY, Zhang LJ, Zhao Q, Liu XJ (2012) Three dimensional CT evaluation of femoral neck anteversion, acetabular anteversion and combined anteversion in unilateral DDH in an early walking age group. Int Orthop 36(1):119-124

2. Mootha Ak, Saini R, Dhillon M, Agarwal S, Wardak E, Kumar V (2010) Do we need femoral derotation osteotomy in DDH of early walking age group? A clinic-radiological correlation study. Arch Orthop Trauma Surg 130:853-858

\footnotetext{
A. K. Mootha $(\bowtie)$

Orthopedics, Siddhartha Hospital,

Kakinada, India

e-mail: akmootha2005@yahoo.com

R. Saini

Orthopedics, Max Hospitals,

Delhi, India
} 